\title{
Colorectal cancer and Blastocystis sp. infection
}

\author{
Violetta Sulżyc-Bielicka ${ }^{*} \mathbb{0}$, Lidia Kołodziejczyk², Małgorzata Adamska³, Bogumiła Skotarczak \\ Sylwia Jaczewska ${ }^{4}$, Krzysztof Safranow ${ }^{5}$, Paweł Bielicki ${ }^{6}$, Józef Kładny ${ }^{7}$ and Dariusz Bielicki ${ }^{8}$
}

\begin{abstract}
Background: Blastocystis sp. is a common intestinal protozoan found worldwide. Based on gene analysis, 17 subtypes (STS, ST1-ST17) have been identified, 9 of which have been isolated from humans. Differences in clinical consequences may depend on differences among the STs. Here, we evaluated the prevalence of Blastocystis sp. in patients with colorectal cancer (CRC) compared to a control group and assessed the relationships between Blastocystis sp. infection and sex; age; and CRC grade, stage, and location.
\end{abstract}

Methods: The study included 107 CRC patients (41 women and 66 men, median age 65 years); 124 subjects without colorectal cancer or a history of oncological disease comprised the control group (55 women and 69 men, median age 63). Stool samples were collected from patients before oncological treatment and examined using light microscopy (iodine-stained smear). Additionally, PCR-based identification of Blastocystis sp. was performed in 95 stool samples from CRC patients and 76 stool samples from the control group.

Results: Light microscopy showed that the prevalence of Blastocystis sp. was significantly higher in CRC patients than in the control group ( $12.15 \%$ and $2.42 \%$, respectively; $p=0.0041)$. Multivariate analysis showed that the odds of Blastocystis sp. infection were fivefold higher in the CRC group than in the control group. PCR-based molecular examinations demonstrated that the proportion of patients infected with Blastocystis sp. was significantly higher in the CRC group than in the control group (12.63\% and $2.63 \%$, respectively; $p=0.023)$. The predominant ST in the CRC group was ST3, detected in nine patients (75\%), followed by ST1 (2 patients, 16.7\%) and ST2 (1 patient, 8.3\%). No association was found between Blastocystis sp. infection and age, sex, or CRC stage, grade, or location.

Conclusions: The results showed that CRC was associated with an increased risk of opportunistic Blastocystis sp. infection, even before oncological treatment. To the best of our knowledge, this is the first report estimating the prevalence of Blastocystis sp. infection in CRC patients before oncological treatment in Europe.

Keywords: Colorectal cancer, Blastocystis sp., ST subtypes, PCR, Light microscopy

\section{Background}

The International Agency for Research on Cancer has estimated that $16 \%$ of cancer worldwide is caused by infectious factors, including parasites [1]. Colorectal cancer $(\mathrm{CRC})$ is one of the most common neoplasms in

*Correspondence: violabielicka@icloud.com

1 Department of Clinical Oncology, Pomeranian Medical University, Szczecin, Poland

Full list of author information is available at the end of the article humans. Most CRCs are sporadic, and the contributions of environmental risk factors have been widely investigated [2]. Microbes colonizing the gut are also considered potential cancer risk factors $[3,4]$.

Blastocystis sp. is a common parasitic protozoan with a worldwide distribution that is found in the gastrointestinal (GI) tract of humans and a wide range of animal hosts $[5,6]$. Its prevalence in humans is estimated to be as high as $10 \%$ in developed countries and $50-60 \%$ in developing countries [7]. Blastocystis sp. is transmitted through 
the faecal-oral route as well as through contaminated water and food $[8,9]$. The pathogenicity of this protozoan is controversial, as it causes non-specific digestive tract symptoms, such as abdominal pain, nausea, vomiting, anorexia, acute or chronic diarrhoea, and weight loss. Blastocystis sp. infection is usually associated with alternating episodes of diarrhoea, normal defecation, and even constipation [10]. Poirier et al. suggested an association between Blastocystis sp. infection and irritable bowel syndrome (IBS) [11]. On the other hand, a higher rate of Blastocystis sp. infection in asymptomatic patients than in those with IBS symptoms was detected in Denmark [12].

The clinical significance of Blastocystis sp. infection remains uncertain, mainly because of its common occurrence in both dyspeptic patients and healthy individuals $[9,13]$. Some studies have maintained that Blastocystis sp. is part of a healthy gut microbiome [13, 14]. However, it has also been reported that Blastocystis sp. infection can have features of opportunistic infection, as has been observed in patients with CRC treated with chemotherapy [15].

The identification of this organism at the species level is difficult. Blastocystis was originally named B. hominis, but subsequent phylogenetic studies limited the name to "Blastocystis species" because of the genetic diversity among members within the genus [16]. It was discovered that host specificity and the pathogenic potential of different isolates are correlated with sequence variations in the small subunit ribosomal RNA (SSU-rRNA) gene [17]. Based on these variations, members of the genus have been ordered into several subtypes (STs) [18]. Based on SSU-rRNA gene analysis, 17 STs (ST1-ST17) have been identified, 9 of which have been isolated from humans [19]. Differences in clinical consequences may depend on differences among STs [20]. Because Blastocystis sp. are found in both symptomatic and asymptomatic patients, the pathogenicity of this organism remains unclear [2123]. Some studies have shown an association between Blastocystis sp. ST variation and pathogenicity. Dogruman-Al et al. [24] suggested that ST2 is a non-pathogenic genotype of Blastocystis sp.

The predominance of Blastocystis sp. ST3 among patients with chronic GI illness has been shown in Malaysia [25], Singapore [26], Egypt [27], Turkey [28], the United States [29], and Iran [30]. Khademvatan et al. [20] showed that in southern Iran, the most common ST of Blastocystis sp. was ST3, which correlated with the presence of GI symptoms in $44.83 \%$ of cases.

Studies evaluating the prevalence of Blastocystis sp. in the French population (inhomogeneous population of 788 patients from 11 hospitals) showed that the frequency of Blastocystis sp. infection in patients with symptoms of GI disorders was not significantly higher than that in patients without symptoms, and the most common ST was ST3 [31].

The aim of this study was to evaluate the prevalence of Blastocystis sp. in patients with CRC compared to that in a control group without colorectal cancer or a history of oncological disease and to assess the relationship of Blastocystis sp. infection with the sex and age of the subjects as well as CRC stage, grade, and location. To the best of our knowledge, this is the first report estimating the prevalence of Blastocystis sp. infection in CRC patients before oncological treatment in Europe.

\section{Methods \\ Patients}

This study included 107 consecutive patients with CRC treated between 2009 and 2014 in the Department of General and Oncological Surgery at Pomeranian Medical University (Szczecin, Poland). There were 41 women $(38.3 \%)$ and 66 men $(61.7 \%)$ in the study group, with a median age of 65 years. The control group comprised 124 individuals without CRC or other neoplasms in their medical history, including 55 women (44.4\%) and 69 men (55.6\%), with a median age of 63 years. Detailed patient characteristics are presented in Table 1. CRC was diagnosed based on colonoscopy examinations conducted in the Department of Gastroenterology at Pomeranian Medical University. Histopathological confirmation of cancer was obtained for all CRC patients. Patients with concomitant neoplasms or with a history of another cancer were excluded from the study. No patient included in the study had previously undergone chemotherapy. Stool samples were taken from CRC patients on the day of admission to the Department of General and Oncological Surgery (before surgery) and were delivered to the Department of Biology and Medical Parasitology at Pomeranian Medical University. The study did not require input from the Bioethics Committee, but the study was approved by the Bioethics Committee of Pomeranian Medical University (No. KB-0012/238/06/18). According to the Bioethics Committee instructions, verbal consent was obtained from patients because of the non-invasive nature of the study. Attestation statements of verbal consent of all participants in the study signed by the physicians were documented in medical records.

\section{Light microscopy (LM)}

Parasitological diagnosis was performed by coproscopy. For the detection of Blastocystis sp., the stool samples were examined using iodine-stained smears ( $\times 40$ magnification). 
Table 1 Characteristics of the CRC patients diagnosed with Blastocystis sp. infection using light microscopy (LM) $(n=107)$ and PCR $(n=95)$

\begin{tabular}{|c|c|c|}
\hline Examined parameters & $\begin{array}{l}\mathrm{LM} \\
n=107\end{array}$ & $\begin{array}{l}\text { PCR } \\
n=95\end{array}$ \\
\hline Age (years) & 65 & 66 \\
\hline Median (range) & $(38-88)$ & $(40-88)$ \\
\hline \multicolumn{3}{|c|}{ Sex, number of patients (\%) } \\
\hline Women & $41(38.3 \%)$ & 37 \\
\hline Men & $66(61.7 \%)$ & 58 \\
\hline \multicolumn{3}{|c|}{ Tumour location*, number (\%) } \\
\hline Right side & $24(22.4 \%)$ & 22 \\
\hline Left side & $83(77.6 \%)$ & 73 \\
\hline \multicolumn{3}{|c|}{ Tumour location*, number (\%) } \\
\hline Rectum & $45(42.1 \%)$ & 40 \\
\hline Colon & $62(57.9 \%)$ & 55 \\
\hline \multicolumn{3}{|l|}{ TNM stage, number (\%) } \\
\hline । & $18(16.8 \%)$ & 18 \\
\hline$\| \mathrm{A}$ & $36(33.7 \%)$ & 33 \\
\hline$\| \mathrm{B}$ & 0 & 0 \\
\hline$\| \mathrm{C}$ & $1(0.9 \%)$ & 0 \\
\hline$\| \mathrm{A}$ & $1(0.9 \%)$ & 1 \\
\hline$\| \mathrm{II}$ & $28(26.2 \%)$ & 23 \\
\hline$\| I I C$ & $7(6.5 \%)$ & 6 \\
\hline IV & $16(15 \%)$ & 14 \\
\hline \multicolumn{3}{|c|}{ Astler-Coller stage, number (\%) } \\
\hline A & $3(2,8 \%)$ & 3 \\
\hline B1 & $15(14 \%)$ & 15 \\
\hline B2 & $36(33.6 \%)$ & 33 \\
\hline B3 & $1(0.9 \%)$ & 0 \\
\hline C1 & $2(1.9 \%)$ & 1 \\
\hline $\mathrm{C} 2$ & $32(29.9 \%)$ & 28 \\
\hline C3 & $2(1.9 \%)$ & 1 \\
\hline D & $16(15 \%)$ & 14 \\
\hline \multicolumn{3}{|c|}{ Astler-Coller stage, number (\%) } \\
\hline$A+B$ & $55(51.4 \%)$ & 51 \\
\hline$C+D$ & $52(48.6 \%)$ & 44 \\
\hline \multicolumn{3}{|l|}{ Grade, number (\%) ${ }^{* *}$} \\
\hline G1 & $7(6.7 \%)$ & 6 \\
\hline G2 & $78(74.3 \%)$ & 68 \\
\hline G3 & $14(13.3 \%)$ & 14 \\
\hline Mucinosum & $6(5.7 \%)$ & 6 \\
\hline \multicolumn{3}{|l|}{ Grade, number $(\%)^{* *}$} \\
\hline $\mathrm{G} 1+\mathrm{G} 2$ & $85(81 \%)$ & 74 \\
\hline G3 + mucinosum & $20(19 \%)$ & 20 \\
\hline
\end{tabular}

*Right-side location including the caecum, colon ascendens, and colon transversum. Left-side location including the colon descendens, sigmoid, and rectum. * *ack of grading ( $\mathrm{G}$ feature) in two patients

\section{Polymerase chain reaction (PCR) analysis}

Among the patients with CRC $(n=107)$, PCR examinations of the stool samples for the presence of Blastocystis
DNA were performed in 95 patients (37 women and 58 men) with a median age of 66 years. Among the control group $(n=124)$, PCR examinations were conducted in 76 patients ( 26 women and 56 men) with a median age of 64 years. The detailed characteristics of patients with CRC who were PCR-tested for the presence of Blastocystis sp. $(n=95)$ are presented in Table 1 . DNA extraction from $200 \mathrm{mg}$ of each stool sample was performed using the QIAamp DNA Stool Kit (Qiagen, Hilden, Germany). Before DNA extraction, three cycles of liquid nitrogen/ water bath $\left(100{ }^{\circ} \mathrm{C}\right)$ incubation, each for $2 \mathrm{~min}$, were performed to destroy the cyst walls of the protozoans present in the samples. Further DNA extraction was conducted according to the manufacturer's instructions. For molecular identification of Blastocystis sp., nested PCR was performed to amplify a $1.1-\mathrm{kb}$ region of the SSU-rRNA gene using two pairs of primers: SR1F and SR1R for the first reaction $[26,32]$ and the Forward B and Reverse B primer set for the second reaction (BöhmGloning et al. [33], modified by Wong et al. [26]). Each amplification reaction was conducted in a total volume of $10 \mu \mathrm{L}$ with the following components: $1 \mu \mathrm{L}$ of DNA, $1 \times$ reaction buffer, $2.5 \mathrm{mM}$ of $\mathrm{MgCl}_{2}, 3 \mathrm{pM}$ of each primer, $0.75 \mathrm{nM}$ of each nucleotide, and $0.5 \mathrm{U}$ of polymerase (Allegro Taq Polymerase, Novazym, Poland). For the first reaction, amplifications were conducted as follows: $3 \mathrm{~min}$ of initial denaturation at $94{ }^{\circ} \mathrm{C}$; followed by 35 cycles of denaturation at $94{ }^{\circ} \mathrm{C}$ for $45 \mathrm{~s}$, annealing at $57^{\circ} \mathrm{C}$ for $45 \mathrm{~s}$, and extension at $72{ }^{\circ} \mathrm{C}$ for $1 \mathrm{~min}$. The PCRs ended with a final extension at $72{ }^{\circ} \mathrm{C}$ for $5 \mathrm{~min}$. For the second reaction, the following conditions were set: initial denaturation at $94{ }^{\circ} \mathrm{C}$ for $3 \mathrm{~min} ; 35$ cycles of denaturation at $94{ }^{\circ} \mathrm{C}$ for $30 \mathrm{~s}$, annealing at $59^{\circ} \mathrm{C}$ for $30 \mathrm{~s}$, and extension at $72{ }^{\circ} \mathrm{C}$ for $45 \mathrm{~s}$; followed by final extension at $72{ }^{\circ} \mathrm{C}$ for $4 \mathrm{~min}$. All PCR amplifications were performed in two replicates in Mastercycler Pro thermal cyclers (Eppendorf, Hamburg, Germany). DNA isolates for positive controls were obtained from stool samples examined previously for the presence of Blastocystis sp. using LM. The PCR products were visualized in $1.5 \%$ agarose gels stained with ethidium bromide. All samples that were positive for the Blastocystis sp. SSU rRNA gene were sequenced (Macrogen, Seoul, Korea) with the Forward B and Reverse B primer sets [26, 33]. The obtained sequences were initially aligned with homologous sequences published in GenBank using BLAST (www.ncbi.nlm.nih.gov) and then using MEGA 6.06 software with ClustalW [34]. Subtype confirmation was performed using the sequence query facility in the Blastocystis $18 \mathrm{~S}$ database (https://pubmlst. org/organisms/blastocystis-spp). 


\section{Statistical analysis}

Associations between Blastocystis sp. infection, and qualitative variables were evaluated with Pearson's chi-square test or Fisher's exact test. Associations of Blastocystis sp. infection with age and rank variables (e.g., cancer stage) were analysed with the Mann-Whitney $U$ test. Multivariate logistic regression analysis was conducted to identify independent risk factors for Blastocystis sp. infection. The threshold for statistical significance was $p<0.05$. Calculations were performed with Statistica 10 (StatSoft Inc., Tulsa, OK, USA) and Microsoft Excel 2003.

\section{Results}

Statistical analysis did not show differences in sex or age between the CRC and control groups (Table 2).

Giardia lamblia was detected in one examined stool sample, and Entamoeba coli was detected in one stool sample. Both cases of parasitic infections were detected in patients with CRC without coexisting Blastocystis sp. infection.

\section{Light microscopy \\ Univariate analysis}

The presence of Blastocystis sp. was detected in 13 patients with CRC (12.15\%) and in 3 individuals in the control group (2.42\%). Blastocystis sp. infection occurred significantly more often in patients with CRC than in individuals in the control group ( $p=0.00409$; Table 2$)$.

\section{Multivariate analysis}

Multivariate logistic regression analysis including Blastocystis sp. infection, CRC diagnosis, age and sex of all participants (CRC and control groups, together $n=107+124)$ as independent variables showed that the odds of Blastocystis sp. infection were fivefold higher in the CRC patients than in the control group (odds ratio [OR] 5.41, 95\% confidence interval [CI] 1.48-19.74, $p=0.010$; Table 3). There was no association between Blastocystis sp. infection and age or sex (Table 3).

\section{PCR analysis \\ Univariate analysis}

Blastocystis sp. infection was detected in 12 patients with CRC (12.63\%) and in two individuals from the control group (2.63\%). Blastocystis sp. detection by PCR occurred significantly more often in patients with CRC than in the control group $(p=0.023)$.

\section{Multivariate analysis}

Multivariate logistic regression analysis including Blastocystis sp. infection, CRC diagnosis, and age and sex of all participants (CRC and control groups) as independent

Table 3 Logistic regression analysis of the association between Blastocystis sp. infection as a dependent variable and sex, age, and CRC diagnosis as independent variables in the CRC and control groups

\begin{tabular}{|c|c|c|c|c|}
\hline \multirow[t]{3}{*}{ Independent variables } & \multicolumn{4}{|c|}{ Blastocystis sp. infection diagnosis method } \\
\hline & \multicolumn{2}{|l|}{ LM } & \multicolumn{2}{|l|}{ PCR } \\
\hline & $\begin{array}{l}\mathrm{OR} \\
95 \% \mathrm{Cl}\end{array}$ & $p$ & $\begin{array}{l}\mathrm{OR} \\
95 \% \mathrm{Cl}\end{array}$ & $p$ \\
\hline Age/1 year of life & $\begin{array}{l}1.0085 \\
0.9588-1.0607\end{array}$ & 0.74 & $\begin{array}{l}0.9891 \\
0.9353-1.0460\end{array}$ & 0.70 \\
\hline Sex & 1.48 & 0.49 & 1.13 & 0.84 \\
\hline Men vs. women & $0.48-4.52$ & & $0.35-3.63$ & \\
\hline CRC & 5.41 & 0.010 & 5.40 & 0.031 \\
\hline CRC vs. control group & $1.48-19.74$ & & $1.16-25.25$ & \\
\hline
\end{tabular}

Blastocystis sp. infection was diagnosed using LM $(n=107+124)$ and PCR analysis $(n=95+76)$

Table 2 Prevalence of Blastocystis sp. in the CRC and control groups determined by LM and PCR

\begin{tabular}{|c|c|c|c|c|c|c|}
\hline \multirow[t]{2}{*}{ Parameter } & \multicolumn{3}{|l|}{ LM } & \multicolumn{3}{|l|}{ PCR } \\
\hline & $\begin{array}{l}\text { Patients with CRC } \\
n=107\end{array}$ & $\begin{array}{l}\text { Control group } \\
n=124\end{array}$ & $p$ & $\begin{array}{l}\text { Patients with CRC } \\
n=95\end{array}$ & $\begin{array}{l}\text { Control group } \\
n=76\end{array}$ & $p$ \\
\hline \multicolumn{7}{|l|}{ Age, years: } \\
\hline Median (range) & $65(38-88)$ & $63(38-88)$ & $0.17^{\mathrm{a}}$ & $66(40-88)$ & $64(38-88)$ & $0.71^{\mathrm{a}}$ \\
\hline \multicolumn{7}{|l|}{ Sex, number (\%) } \\
\hline Women & 41 (38.3\%) & $55(44.3 \%)$ & $0.42^{b}$ & $37 \%$ & $26 \%$ & $0.63^{b}$ \\
\hline Men & $66(61.7 \%)$ & $69(55.7 \%)$ & & $58 \%$ & $50 \%$ & \\
\hline \multicolumn{7}{|c|}{$\begin{array}{l}\text { Blastocystis sp. infection, } \\
\text { patients (\%) }\end{array}$} \\
\hline Present & $13(12.15 \%)$ & $3(2.42 \%)$ & $0.0041^{b}$ & $12(12.63 \%)$ & $2(2.63 \%)$ & 0.02 \\
\hline Absent & $94(87.85 \%)$ & $121(97.58 \%)$ & & 83 (87.37\%) & 74 (97.37\%) & $3^{b}$ \\
\hline
\end{tabular}

\footnotetext{
${ }^{a}$ Mann-Whitney $U$ test
}

b Fisher's exact test 
variables showed that the odds of Blastocystis sp. infection were fivefold higher in the CRC patients than in the control group (OR: 5.40, 95\% CI 1.16-25.25, $p=0.031$; Table 3). There was no association between Blastocystis sp. infection and age or sex (Table 3). The predominant ST among patients with CRC was ST3, which was detected in nine patients (75\%). ST1 was detected in two patients, and ST2 in one patient. ST1 and ST3 were detected in one patient each in the control group (Table 4). The sequences obtained in this study were deposited in the GenBank database under accession numbers MG214872-MG214885. Three of the sequences (accession nos. MG214878, MG214882, and MG214884) had $98.1-100 \%$ similarity to the published sequence JQ665862, representing ST1. One sequence obtained in this study (accession no. MG214880) was identical to the published sequence JQ665848, representing ST2. The remaining 10 sequences (accession nos. MG214872, MG214873, MG214874, MG214875, MG214876, MG214877, MG214879, MG214881, MG214883, and MG214885) had $99.4-100 \%$ similarity to the published sequence KX618192, representing ST3.

\section{Comparison of $L M$ and PCR analysis results}

Among the patients with CRC for whom both LM and PCR were performed for diagnosis of Blastocystis sp. infection $(n=95)$, Blastocystis sp. was detected by LM in 12 patients $(12.6 \%)$, and 9 of those samples $(9.4 \%)$ were confirmed by PCR examination (Table 5). In those samples, sequencing identified ST3 (Table 4). In the remaining three samples, Blastocystis sp. infection detected by LM was not confirmed by PCR. In 3 of 83 stool samples, the presence of Blastocystis sp. was not detected by LM but was detected by PCR ( 2 samples with ST1 and 1 sample with ST2) (Table 4). In all nine samples positive for ST3 from patients with CRC, Blastocystis sp. was also detected using LM. There was a strong positive correlation between the LM and PCR results in the CRC group (phi coefficient $=+0.71$ ).

In the subgroup of controls in which both LM and PCR analysis were performed $(n=76)$, the presence of Blastocystis sp. was detected by LM in one sample (1.3\%), but this was not confirmed by PCR. In two stool samples, Blastocystis sp. presence was detected by PCR (1 sample with ST1 and 1 sample with ST3), but it was not
Table 5 Comparison of the LM and PCR results for the detection of Blastocystis sp. in CRC patients

\begin{tabular}{llll}
\hline LM & \multicolumn{3}{l}{$P C R$} \\
\cline { 2 - 4 } & PCR- (\%) & PCR+ (\%) & Total (\%) \\
\hline LM- (\%) & $80(84 \%)$ & $3(3 \%)$ & $83(87 \%)$ \\
LM+ (\%) & $3(3 \%)$ & $9(10 \%)$ & $12(13 \%)$ \\
Total (\%) & $83(87 \%)$ & $12(13 \%)$ & $95(100 \%)$ \\
\hline
\end{tabular}

detected by LM in either case. Therefore, in the control group, there was no correlation between LM and PCR, as no sample was Blastocystis-positive by both methods $(\mathrm{phi}=-0.02)$. There were also no associations between Blastocystis sp. infection and age, gender, grade, AstlerColler and tumour-node-metastasis (TNM) stages, location of tumour in the rectum compared to the colon, or left- or right-side location of the tumour according to splenic flexion in CRC patients.

\section{Discussion}

Microbiota alterations, referred to as dysbiosis, are often associated with CRC. Both human studies and studies conducted in animals showed that the gut microbiota related to CRC was distinct from that in subjects without CRC [35-37]. In addition, two meta-analyses of faecal metagenome changes specific to CRC were published in $2019[38,39]$. The human gut microbiota comprises bacteria, viruses, and eukaryotes (e.g., protozoa, helminths, and fungi). In human CRC samples, cytomegalovirus (CMV), John Cunningham (JC) virus, and human papillomavirus (HPV) have been identified, although the data are conflicting $[37,40]$. Additionally, changes in the mycobiome associated with human CRC have been reported [41].

The mechanism of the impact of dysbiosis on CRC carcinogenesis encompasses inflammation, immune regulation, metabolism of dietary components, and genotoxin production [42]. The gut microbiota interacting with the host immune system can affect the inflammatory process in the GI tract [43]. The microbiota produce numerous metabolites significant for human physiology [44]; on the other hand, these metabolites can impact the risk of developing CRC.

Table 4 Prevalence of Blastocystis sp. infection detected using LM and PCR with differentiation of STs (CRC group, $n=95 ;$ control group, $n=76$ )

\begin{tabular}{llllll}
\hline & $\mathrm{LM}+(\%)^{*}$ & $\mathrm{PCR}+(\%)^{* *}$ & $\mathrm{ST} 1 \mathrm{n}+(\%)^{* * *}$ & $\mathrm{ST} 2 \mathrm{n}+(\%)^{* * *}$ & $\mathrm{ST} \mathrm{n}+(\%)^{* * *}$ \\
\hline CRC group $(n=95)$ & $12(12.6)$ & $12(12.6)$ & $2(16.7)$ & $1(8.3)$ & $9(75)$ \\
Control group $(n=76)$ & $1(1.3)$ & $2(2.6)$ & $1(50)$ & - & $1(50)$ \\
\hline
\end{tabular}

*Positive results on $\mathrm{LM}$, ${ }^{* *}$ positive results on $\mathrm{PCR}$, ${ }^{* * *}$ positive sample 
Another carcinogenic mechanism of the microbiota is the production of DNA-damaging toxins [40, 45, 46]. In a driver-passenger model of CRC, the mucosa of the colon is colonized by pathogenic driver bacteria, which produce genotoxins that induce inflammation and, consequently, the adenoma-carcinoma sequence [47]. On the other hand, opportunistic passenger bacteria might proliferate in CRC tumours and stimulate the infiltration of immune cells $[40,47]$.

Disruptions in the gut microbiota and changes in its relative abundance can alter the balance, leading to many diseases, including inflammatory bowel disease (IBD) and Clostridium difficile infection [48, 49]. Impairment of the symbiotic relationship between the microbiota and the host leads to immune dysregulation and can induce chronic inflammation, resulting in IBD [48]. The microbiota composition varies between certain subtypes of IBD (Crohn's disease, colitis ulcerosa) and the presence of an active phase [50-52]. The role of gut microbiota alterations in IBD has been widely examined [53, 54]. However, in our study, we focused on patients with CRC, and none of our patients suffered from IBD.

The pathogenic potential of Blastocystis sp. remains controversial [55-57]. Blastocystis sp. interact with bacterial gut microbes $[57,58]$. The increased prevalence of Blastocystis sp. is related to changes in the composition of the microbiota in the human host [59-62]. Modifications of the microbiota affect the host immune response $[55,63]$.

To the best of our knowledge, this is the first report to estimate the prevalence of Blastocystis sp. infection in CRC patients before oncological treatment in Europe. Few studies have examined Blastocystis sp. infection in CRC patients (those that have were from Uzbekistan, Saudi Arabia, Turkey, and Malaysia) [64-67]. Most studies indicate opportunistic characteristics of Blastocystis sp. infection, but there are also reports indicating that Blastocystis sp. is a component of the healthy gut microbiome [14].

Our results were obtained from a homogeneous group of patients with CRC before oncological treatment, and individuals in the control group were matched by age to the CRC patients. The results showed that the odds of Blastocystis sp. infection patients with CRC were 5 times higher than those in the control group; these results were obtained not only by LM but also by PCR, and the proportion of individuals infected with Blastocystis sp. was significantly higher in the CRC group (12.63\%) than in the control group (2.63\%). Chandramathi et al. [15] demonstrated the opportunistic characteristics of Blastocystis sp. infection among patients with CRC $(n=15)$. Our results suggest that $C R C$ is related to an increased risk of opportunistic infection with Blastocystis sp. even before oncological treatment, which may have additional effects on the immunological system.

At the time of planning of our study, we could not predict what the results would be, because Blastocystis sp. can be considered a marker of a healthy gut microbiota [57]; however, it is not possible to exclude opportunistic Blastocystis sp. infection in patients with CRC.

A significantly higher prevalence of Blastocystis sp. (80\%) was found using LM in 200 CRC patients compared to the control population in Tashkent, Uzbekistan [64]. The prevalence of Blastocystis sp. in CRC patients was fourfold higher than that in the control population. However, the authors did not conduct PCR analysis, which could have enabled the determination of the ST of Blastocystis sp. [64]. Yersal et al. [66] detected Blastocystis sp. in $6.5 \%$ of 232 stool samples from cancer patients suffering from different types of cancer (lung, breast, CRC) by LM, but in the CRC patients, Blastocystis sp. was detected using PCR in $7.5 \%$ of the 66 examined cases [66], among which ST1 was the predominant ST (3 cases, $60 \%$ ), followed by ST3 (2 cases, 40\%). In our study, ST3 was the predominant ST in CRC patients (9 patients, 75\%), followed by ST1 (2 patients, 16.7\%) and ST2 (1 patient, 8.3\%).

Kumarasamy et al. found Blastocystis sp. infection prevalence rates of $22.08 \%$ in 204 Malaysian CRC patients and $9.95 \%$ in the control group [67]. The most common ST was ST3 (12.75\%), followed by ST1 (4.41\%), ST2 (0.49\%), and ST5 (0.49\%) [67].

Blastocystis sp. infection was confirmed in 74 CRC patients (29.7\%) in Saudi Arabia [65], where ST1 was the most predominantly detected ST $(54,5 \%)$ with a significant risk association (crude OR: 7.548; 95\% CI 1.62934.987; $p=0.004$ ).

On the other hand, Beghini et al. [14] showed a lower frequency of Blastocystis sp. infection among patients with CRC (5.7\%) compared to the healthy control group in their analysis of 12 studies of patient populations from different continents $(n=1689)$ with different diseases of the GI tract, including CRC. It is worth noting that only 53 of those patients suffered from CRC $[3,14]$.

Differences in the results of studies on the prevalence of various Blastocystis sp. STs may be related to the genetic diversity of Blastocystis sp., non-homogeneity of the analysed patient groups, and ethnic diversity among the examined populations inhabiting different parts of the world [56].

A possible role for Blastocystis sp. in CRC pathogenesis has been suggested $[15,64,68,69]$. Postulated potential carcinogenic effects of Blastocystis sp. infection in humans, especially CRC patients, was examined by Chan et al. [70], and the ability of Blastocystis sp. to induce the growth of CRC cell lines by inhibiting the apoptotic 
effects of CRC cells has been documented [70]. Furthermore, isolated antigens of Blastocystis sp. isolates were shown to promote the proliferation of cancer cells via downregulation of host immune cellular responses [22, 68, 70]. Chan et al. [70] showed that antigens isolated from symptomatic human hosts caused a more extensive inflammatory reaction and a higher proliferation rate of CRC cells than isolates from asymptomatic human hosts. Chandramathi et al. [68] also showed that solubilized antigen of Blastocystis sp. facilitates growth in the human HCT116 CRC cell line. The antigen isolated from ST3 had the most prominent effect on the proliferation of CRC cells [71], which confirmed the case of severe ST3 Blastocystis infection in a 35-year-old man at the time of CRC diagnosis, as described by Padukone et al. [72].

The possible impact of Blastocystis sp. infections on CRC carcinogenesis remains unclear. The pathogenicity of Blastocystis sp. is suspected to be caused by the release of cysteine proteases by this protozoan. These proteases stimulate mucosal cells to release interleukin 8 , which has been associated with gut inflammation [56].

Chronic inflammation is an established risk factor for CRC [73]. Among the molecular mechanisms of CRC pathogenesis, oxidative stress plays an important role and has been shown to be associated with Blastocystis sp. infection [69].

In our study, Blastocystis sp. infection was confirmed by both LM and PCR in all nine samples positive for ST3 from patients with CRC, but no such confirmation was found for the ST1 or ST2 genotypes. In three CRC patients, Blastocystis sp. infection detected by LM was not confirmed by PCR, possibly due to the presence of other organisms in the stool samples and non-specific amplification, which was revealed by sequencing of the obtained PCR products. A possible explanation for this discrepancy could also be a misdiagnosis of the parasite by light microscopy.

However, there was a strong positive correlation between the LM and PCR results in the CRC group (phi coefficient $=+0.71$ ). The presented results show that LM is an inexpensive, sensitive, and accessible method in daily practice. On the other hand, the PCR method allows for the identification of STs that may have different pathogenic potentials.

\section{Conclusions}

We demonstrated that the prevalence of Blastocystis sp. was higher in CRC patients than in the control group, independent of the diagnostic method used. In addition, ST3 was the predominant Blastocystis sp. ST among Polish CRC patients. Furthermore, Blastocystis sp. infection occurred five times more often in the CRC group than in the control group, independent of age, sex, and diagnostic method. No association was found between Blastocystis sp. infection and age, sex, staging, grading, or CRC location. Together, these results show that CRC is associated with an increased risk of opportunistic Blastocystis sp. infection even before oncological treatment. The potential relationship of Blastocystis sp. with CRC carcinogenesis needs further study (Additional file 1).

\section{Supplementary Information}

The online version contains supplementary material available at https://doi. org/10.1186/s13071-021-04681-x.

Additional file 1. Database.

\section{Acknowledgements}

The authors gratefully acknowledge Marek Sawczuk from the Department of Genetics, Faculty of Biology, University of Szczecin, for excellent assistance with the PCR analysis. We are grateful to the study subjects for their participation.

\section{Authors' contributions}

Conceptualisation: VS-B, LK. Data curation: VS-B, LK, KS, SJ, PB. Formal analysis: VS-B, LK, MA, BS, PB, KS. Funding acquisition: VS-B. Investigation: MA, BS, LK, VS-B, DB. Methodology: MA, BS, LK. Project administration: VS-B. Resources: JK, DB. Software: KS. Supervision: VS-B, LK, DB. Validation: LK, MA, BS. Visualisation: KS. Writing —original draft: VS-B, LK, MA, BS, PB. Writing-review \& editing: VS-B, LK, MA, BS, PB. All authors read and approved the final manuscript.

\section{Funding}

This work was supported by Pomeranian Medical University, grant number WLBiML-418-02/S/12/2019, https://www.pum.edu.pl. The funders had no role in the study design, data collection and analysis, decision to publish, or preparation of the manuscript.

\section{Availability of data and materials}

All data generated or analysed during this study are included in this published article and attached in the "Sulzyc-Bielicka-database xIs" file.

\section{Declarations}

\section{Ethics approval and consent to participate}

All procedures were reviewed and approved by the Bioethics Committee of Pomeranian Medical University (No. KB-0012/238/06/18). According to the Bioethics Committee instructions, verbal consent was obtained from patients because of non-invasive nature of the conducted study. Examinations were performed in addition to routine stool sample investigations for parasite infection. Attestation statements of verbal consent of all participants in the conducted study signed by the physicians were documented in medical records.

\section{Consent for publication}

Not applicable.

\section{Competing interests}

The authors declare that they have no competing interests.

\section{Author details}

${ }^{1}$ Department of Clinical Oncology, Pomeranian Medical University, Szczecin, Poland. ${ }^{2}$ Department of Biology and Medical Parasitology, Pomeranian Medical University, Szczecin, Poland. ${ }^{3}$ Institute of Biology, University of Szczecin, Szczecin, Poland. ${ }^{4}$ Early Phase Clinical Trial Unit. Maria Sklodowska-Curie Memorial Cancer Centre-Institute of Oncology, Warsaw, Poland. ${ }^{5}$ Department of Biochemistry and Medical Chemistry, Pomeranian Medical University, Szczecin, Poland. ${ }^{6}$ Department of Radiotherapy, Pomeranian Medical University, Szczecin, Poland. ${ }^{7}$ Department of Oncological Surgery, Pomeranian Medical 
University, Szczecin, Poland. ${ }^{8}$ Department of Gastroenterology, Pomeranian Medical University, Szczecin, Poland.

Received: 15 September 2020 Accepted: 15 March 2021 Published online: 14 April 2021

\section{References}

1. Parkin DM. The global health burden of infection-associated cancers in the year 2002. Int J Cancer. 2006;118:3030-44.

2. Lichtenstein P, Holm NV, Verkasalo PK, Iliadou A, Kaprio J, Koskenvuo M, et al. Environmental and heritable factors in the causation of canceranalyses of cohorts of twins from Sweden, Denmark, and Finland. N Engl J Med. 2000;343:78-85.

3. Zeller G, Tap J, Voigt AY, Sunagawa S, Kultima JR, Costea PI, et al. Potential of fecal microbiota for early-stage detection of colorectal cancer. Mol Syst Biol. 2014;10:766.

4. Jahani-Sherafat S, Alebouyeh M, Moghim Sh, Ahmadi-Amoli H, Ghasemian Safaei $\mathrm{H}$. Role of gut microbiota in the pathogenesis of colorectal cancer; a review article. Gastroenterol Hepatol Bed Bench. 2018;11:101-9.

5. Alfellani MA, Jacob AS, Perea NO, Krecek RC, Taner-Mulla D, Verweij JJ, et al. Diversity and distribution of Blastocystis sp. subtypes in non-human primates. Parasitology. 2013;140:966-71.

6. Menounos PG, Spanakos G, Tegos N, Vassalos CM, Papadopoulou C, Vakalis NC. Direct detection of Blastocystis sp. in human faecal samples and subtype assignment using single strand conformational polymorphism and sequencing. Mol Cell Probes. 2008;22:24-9.

7. Tan KS. Blastocystis in humans and animals: new insights using modern methodologies. Vet Parasitol. 2004;126:121-44.

8. Leelayoova S, Siripattanapipong S, Thathaisong U, Naaglor T, Taamasri P, Piyaraj P, Mungthin M. Drinking water: a possible source of Blastocystis spp. subtype 1 infection in schoolchildren of a rural community in central Thailand. Am J Trop Med Hyg. 2008;79:401-6.

9. Ramirez JD, Sánchez LV, Bautista DC, Corredor AF, Flórez AC, Stensvold CR. Blastocystis subtypes detected in humans and animals from Colombia. Infect Genet Evol. 2014;22:223-8.

10. Rene BA, Stensvold CR, Badsberg JH, Nielsen HV. Subtype analysis of Blastocystis isolates from Blastocystis cyst excreting patients. Am J Trop Med Hyg. 2009;80:588-92.

11. Poirier P, Wawrzyniak I, Vivarès CP, Delbac F, El Alaoui H. New insights into Blastocystis spp.: a potential link with irritable bowel syndrome. PLoS Pathog. 2012;8:

12. Krogsgaard LR, Engsbro AL, Stensvold CR, Nielsen HV, Bytzer P. The prevalence of intestinal parasites is not greater among individuals with irritable bowel syndrome: a Population-based Case-control Study. Clin Gastroenterol Hepatol. 2015;13(507-13):

13. Scanlan PD, Stensvold CR, Rajilić-Stojanović M, Heilig HG, De Vos WM, O'Toole PW, Cotter PD. The microbial eukaryote Blastocystis is a prevalent and diverse member of the healthy human gut microbiota. FEMS Microbiol Ecol. 2014;90:326-30.

14. Beghini F, Pasolli E, Truong TD, Putignani L, Cacciò SM, Segata N. Largescale comparative metagenomics of Blastocystis, a common member of the human gut microbiome. ISME J. 2017;11:2848-63.

15. Chandramathi S, Suresh K, Anita ZB, Kuppusamy UR. Infections of Blastocystis hominis and microsporidia in cancer patients: are they opportunistic? Trans R Soc Trop Med Hyg. 2012;106:267-9.

16. Stensvold CR, Suresh GK, Tan KS, Thompson RC, Traub RJ, Viscogliosi E, Yoshikawa H, Clark CG. Terminology for Blastocystis subtypes - a consensus. Trends Parasitol. 2007;23:93-6.

17. Noel C, Dufernez F, Gerbod D, Edgcomb VP, Delgado-Viscogliosi P, Ho LC. Molecular phylogenies of Blastocystis isolates from different hosts: implications for genetic diversity, identification of species, and zoonosis. J Clin Microbiol. 2005;43:348-55.

18. Stensvold CR. Blastocystis: genetic diversity and molecular methods for diagnosis and epidemiology. Trop Parasitol. 2013;3:26-34.

19. Stensvold CR, Clark CG. Current status of Blastocystis: a personal view. Parasitol Int. 2016;65:763-71.

20. Khademvatan S, Masjedizadeh R, Yousefi-Razin E, Mahbodfar H, Rahim F, Yousefi E, Foroutan M. PCR-based molecular characterization of
Blastocystis hominis subtypes in southwest of Iran. J Infect Public Health. 2018;11:43-7.

21. Khademvatan S, Masjedizadeh R, Rahim F, Mahbodfar H, Salehi R, YousefiRazin E, Foroutan M. Blastocystis and irritable bowel syndrome: frequency and subtypes from Iranian patients. Parasitol Int. 2017;66:142-5.

22. Ajjampur SSR, Tan KSW. Pathogenic mechanisms in Blastocystis spp. - Interpreting results from in vitro and in vivo studies. Parasitol Int. 2016:65:772-9.

23. Andersen LO, Stensvold C. Blastocystis in health and disease: are we moving from a clinical to a public health perspective? J Clin Microbiol. 2016;54:524-8.

24. Dogruman-Al F, Dagci H, Yoshikawa H, Kurt O, Demirel M. A possible link between subtype 2 and asymptomatic infections of Blastocystis hominis. Parasitol Res. 2008;103:685-9.

25. Tan TC, Suresh KG, Smith HV. Phenotypic and genotypic characterisation of Blastocystis hominis isolates implicates subtype 3 as a subtype with pathogenic potential. Parasitol Res. 2008;104:85-93.

26. Wong KH, Ng GC, Lin RT, Yoshikawa H, Taylor MB, Tan KS. Predominance of subtype 3 among Blastocystis isolates from a major hospital in Singapore. Parasitol Res. 2008;102:663-70.

27. Hussein EM, Hussein AM, Eida MM, Atwa MM. Pathophysiological variability of different genotypes of human Blastocystis hominis Egyptian isolates in experimentally infected rats. Parasitol Res. 2008;102:853-60.

28. Ozyurt M, Kurt O, Mølbak K, Nielsen HV, Haznedaroglu T, Stensvold CR. Molecular epidemiology of Blastocystis infections in Turkey. Parasitol Int 2008;57:300-6.

29. Jones MS, Whipps CM, Ganac RD, Hudson NR, Boorom K. Association of Blastocystis subtype 3 and 1 with patients from an Oregon community presenting with chronic gastrointestinal illness. Parasitol Res. 2009; 104:341-5.

30. Moosavi A, Haghighi A, Mojarad EN, Zayeri F, Alebouyeh M, Khazan H, Kazemi B, Zali MR. Genetic variability of Blastocystis sp. isolated from symptomatic and asymptomatic individuals in Iran. Parasitol Res. 2012;111:2311-5

31. ElSafadi D, Cian A, Nourrisson C, Pereira B, Morelle C, Bastien P, et al. Prevalence, risk factors for infection and subtype distribution of the intestinal parasite Blastocystis sp. from a large-scale multi-center study in France. BMC Infect Dis. 2016;16:451.

32. Yoshikawa H, Abe N, Iwasawa M, Kitano S, Nagano I, Wu Z, Takahashi Y. Genomic analysis of Blastocystis hominis strains isolated from two longterm health care facilities. J Clin Microbiol. 2000;38:1324-30.

33. Böhm-Gloning B, Knobloch J, Walderich B. Five subgroups of Blastocystis hominis isolates from symptomatic and asymptomatic patients revealed by restriction site analysis of PCR-amplified 16S-like rDNA. Trop Med Int Health. 1997;2:771-8.

34. Tamura K, Stecher G, Peterson D, Filipski A, Kumar S. MEGA6: molecular evolutionary genetics analysis version 60. Mol Biol Evol. 2013;28:2731-9.

35. Castellarin M, Warren RL, Freeman JD, Dreolini L, Krzywinski M, Strauss J, et al. Fusobacterium nucleatum infection is prevalent in human colorectal carcinoma. Genome Res. 2012;22:299-306.

36. Feng $Q$, Liang $S$, Jia H, Stadlmayr A, Tang L, Lan Z, et al. Gut microbiome development along the colorectal adenoma-carcinoma sequence. Nat Commun. 2015:6:6528.

37. Yu J, Feng Q, Wong SH, Zhang D, Liang Q, Qin Y, Tang L, Zhao H, Stenvang J, Li Y, Wang X. Metagenomic analysis of faecal microbiome as a tool towards targeted non-invasive biomarkers for colorectal cancer. Gut. 2017;66:70-8.

38. Thomas AM, Manghi P, Asnicar F, Pasolli E, Armanini F, Zolfo M, et al. Metagenomic analysis of colorectal cancer datasets identifies crosscohort microbial diagnostic signatures and a link with choline degradation. Nat Med. 2019;25:667-78.

39. Wirbel J, Pyl PT, Kartal E, Zych K, Kashani A, Milanese A, et al. Meta-analysis of fecal metagenomes reveals global microbial signatures that are specific for colorectal cancer. Nat Med. 2019;25:679-89.

40. Wong SH, Yu J. Gut microbiota in colorectal cancer: mechanisms of action and clinical applications. Nat Rev Gastroenterol Hepatol. 2019;16:690-704

41. Coker OO, Nakatsu G, Dai RZ, Wu WKK, Wong SH, Ng SC, et al. Enteric fungal microbiota dysbiosis and ecological alterations in colorectal cancer. Gut. 2019;68:654-62. 
42. Tilg $H$, Adolph TE, Gerner RR, Moschen AR. The intestinal microbiota in colorectal cancer. Cancer Cell. 2018;33:954-64.

43. Tomkovich S, Yang Y, Winglee K, Gauthier J, Mühlbauer M, Sun X, et al. Locoregional Effects of Microbiota in a Preclinical Model of Colon Carcinogenesis. Cancer Res. 2017;77:2620-32.

44. Louis P, Hold GL, Flint HJ. The gut microbiota, bacterial metabolites and colorectal cancer. Nat Rev Microbiol. 2014;12:661-72.

45. He Z, Gharaibeh RZ, Newsome RC, Pope JL, Dougherty MW, Tomkovich $\mathrm{S}$, et al. Campylobacter jejuni promotes colorectal tumorigenesis through the action of cytolethal distending toxin. Gut. 2019;68:289-300.

46. Cougnoux A, Delmas J, Gibold L, Faïs T, Romagnoli Ch, Robin F, et al. Small-molecule inhibitors prevent the genotoxic and protumoural effects induced by colibactin-producing bacteria. Gut. 2016;65:278-85.

47. Tjalsma H, Boleij A, Marchesi JR, Dutilh BE. A bacterial driver-passenger model for colorectal cancer: beyond the usual suspects. Nat Rev Microbiol. 2012;10:575-82.

48. Kostic AD, Xavier RJ, Gevers D. The microbiome in inflammatory bowel disease: current status and the future ahead. Gastroenterology. 2014;146:1489-99.

49. Buffie CG, Bucci V, Stein RR, McKenney PT, Ling L, Gobourne A, et al. Precision microbiome reconstitution restores bile acid mediated resistance to Clostridium difficile. Nature. 2015;517:205-8.

50. Pascal V, Pozuelo M, Borruel N, Casellas F, Campos D, Santiago A, et al. A microbial signature for Crohn's disease. Gut. 2017;66:813-22.

51. Machiels K, Joossens M, Sabino J, De Preter V, Arijs I, Eeckhaut V, et al. A decrease of the butyrate-producing species Roseburia hominis and Faecalibacterium prausnitzii defines dysbiosis in patients with ulcerative colitis. Gut. 2014;63:1275-83.

52. Hold GL, Smith M, Grange C, Watt ER, El-Omar EM, Mukhopad-hya I. Role of the gut microbiota in inflammatory bowel disease pathogenesis: What have we learnt in the past 10 years? World J Gastroenterol. 2014;20:1192-210.

53. Prosberg M, Bendtsen F, Vind I, Petersen AM, Gluud LL. The association between the gut microbiota and the inflammatory bowel disease activity: a systematic review and meta-analysis. Scand J Gastroenterol. 2016;51:1407-15

54. Sankarasubramanian J, Ahmad R, Avuthu N, Singh AB, Guda C. Gut microbiota and metabolic specificity in ulcerative colitis and Crohn's disease. Front Med. 2020;7:

55. Partida-Rodríguez O, Serrano-Vázquez A, Nieves-Ramírez ME, Moran $P$, Rojas L, Portillo T, et al. Human intestinal microbiota: interaction between parasites and the host immune response. Arch Med Res. 2017;48:690-700.

56. Kumarasamy $\vee$, Anbazhagan D, Subramaniyan $\bigvee$, Vellasamy S. Blastocystis sp., Parasite Associated with Gastrointestinal Disorders: An Overview of its Pathogenesis, Immune Modulation and Therapeutic Strategies. Curr Pharm Des. 2018;24:3172-5.

57. Stensvold CR, van der Giezen M. Associations between Gut Microbiota and Common Luminal Intestinal Parasites. Trends Parasitol. 2018;34:369-77

58. Kök M, Çekin Y, Çekin AH, Uyar S, Harmandar F, Şahintürk Y. The role of Blastocystis hominis in the activation of ulcerative colitis. Turk J Gastroenterol. 2019:30:40-6.
59. Yildiz S, Doğan I, Doğruman-AI F, Nalbantoğlu U, Üstek D, Sarzhanov F, Yildirim S. Association of Enteric Protist Blastocystis spp and Gut Microbiota with Hepatic Encephalopathy. J Gastrointestin Liver Dis. 2016;25:489-97.

60. Engsbro AL, Stensvold CR, Vedel Nielsen H, Bytzer P. Prevalence, incidence, and risk factors of intestinal parasites in Danish primary care patients with irritable bowel syndrome. Scand J Infect Dis. 2014;46:204-9.

61. Nourrisson C, Scanzi J, Pereira B, et al. Blastocystis is associated with decrease of fecal microbiota protective bacteria: comparative analysis between patients with irritable bowel syndrome and control subjects. PLOS ONE. 2014;9:

62. lebba V, Santangelo F, Totino V, Pantanella F, Monsia A, DiCristanziano V, et al. Gut microbiota related to Giardia duodenalis, Entamoeba spp and Blastocystis hominis infections in humans from Cote d'Ivoire. J Infect Dev Ctries. 2016;10:

63. Berrilli F, Di Cave D, Cavallero S, Cavallero S, D'Amelio S. Interactions between parasites and microbial communities in the human gut. Front Cell Infect Microbiol. 2012;2:141.

64. Toychiev A, Abdujapparov S, Imamov A, Navruzov B, Davis N, Badalova $\mathrm{N}$, Osipova S. Intestinal helminths and protozoan infections in patients with colorectal cancer: prevalence and possible association with cancer pathogenesis. Parasitol Res. 2018;117:3715-23.

65. Mohamed AM, Ahmed MA, Ahmed SA, Al-Semany SA, Alghamdi SS, Zaglool DA. Predominance and association risk of Blastocystis hominis subtype I in colorectal cancer: a case control study. Infect Agent Cancer. 2017;12:21

66. Yersal O, Malatyali E, Ertabaklar H, Oktay E, Barutca S, Ertug S. Blastocystis subtypes in cancer patients: analysis of possible risk factors and clinical characteristics. Parasitol Int. 2016;65:792-6.

67. Kumarasamy V, Roslani AC, Rani KU, Kumar Govind S. Advantage of using colonic washouts for Blastocystis detection in colorectal cancer patients. Parasit Vectors. 2014;7:162.

68. Chandramathi S, Suresh K, Kuppusamy UR. Solubilized antigen of Blastocystis hominis facilitates the growth of human colorectal cancer cells, HCT116. Parasitol Res. 2010;106:941-5.

69. Kumarasamy V, Kuppusamy UR, Jayalakshmi P, Samudi C, Ragavan ND, Kumar S. Exacerbation of colon carcinogenesis by Blastocystis sp. PLoS ONE. 2017;12:

70. Chan KH, Chandramathi S, Suresh K, Chua KH, Kuppusamy UR. Effects of symptomatic and asymptomatic isolates of Blastocystis hominis on colorectal cancer cell line, HCT116. Parasitol Res. 2012;110:2475-80.

71. Kumarasamy V, Kuppusamy UR, Samudi C, Kumar S. Blastocystis sp. subtype 3 triggers higher proliferation of human colorectal cancer cells, HCT116. Parasitol Res. 2013;112:3551-5.

72. Padukone S, Mandal J, Parija SC. Severe Blastocystis subtype 3 infection in a patient with colorectal cancer. Trop Parasitol. 2017;7:122-4.

73. Lasry A, Zinger A, Ben-Neriah Y. Inflammatory networks underlying colorectal cancer. Nat Immunol. 2016;17:230-40.

\section{Publisher's Note}

Springer Nature remains neutral with regard to jurisdictional claims in published maps and institutional affiliations.
Ready to submit your research? Choose BMC and benefit from:

- fast, convenient online submission

- thorough peer review by experienced researchers in your field

- rapid publication on acceptance

- support for research data, including large and complex data types

- gold Open Access which fosters wider collaboration and increased citations

- maximum visibility for your research: over 100M website views per year

At BMC, research is always in progress.

Learn more biomedcentral.com/submissions 